Supporting Information:

\title{
Lithography-Free Formation of Nanopores in Plastic Membranes using Laser Heating
}

\author{
Shanshan Wu, Sang Ryul Park and Xinsheng Sean Ling* \\ Department of Physics, Brown University, Providence, Rhode Island 02912, USA
}

*Corresponding Author: Prof. Xinsheng Sean Ling,

Brown University, 182 Hope Street, Providence, Rhode Island 02912 USA

Tel: (401)-863-2582; Email: Xinsheng_Ling@brown.edu 


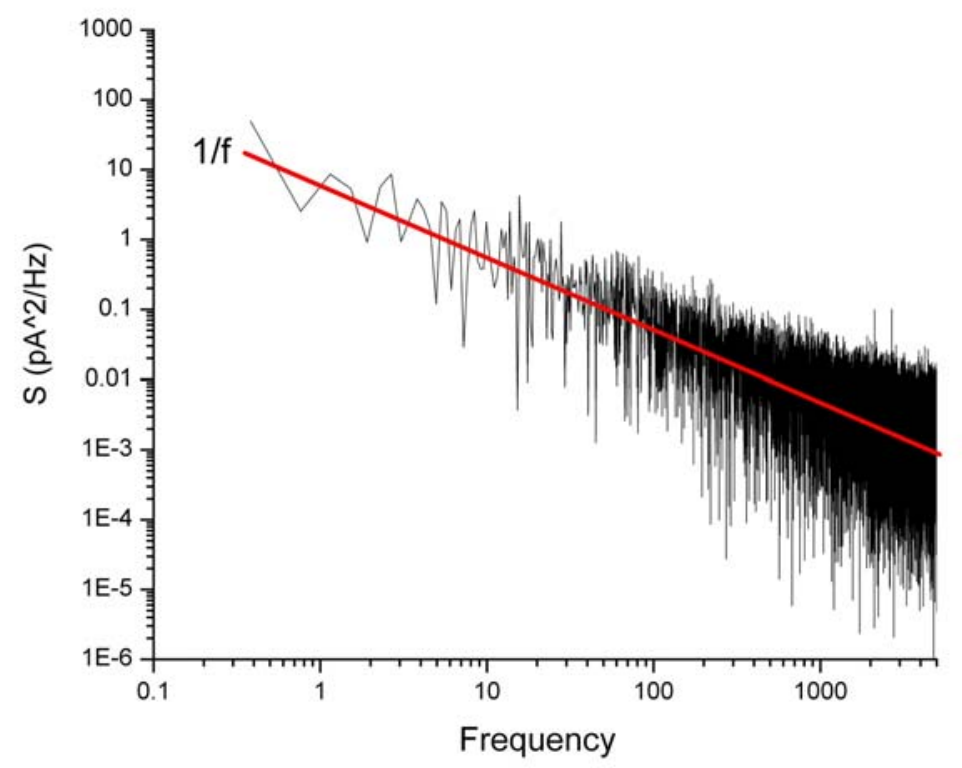

Supporting Figure 1. Power spectrum of the open-pore current noise of a wax nanopore. The red line indicates $1 / \mathrm{f}$ behavior. 
a

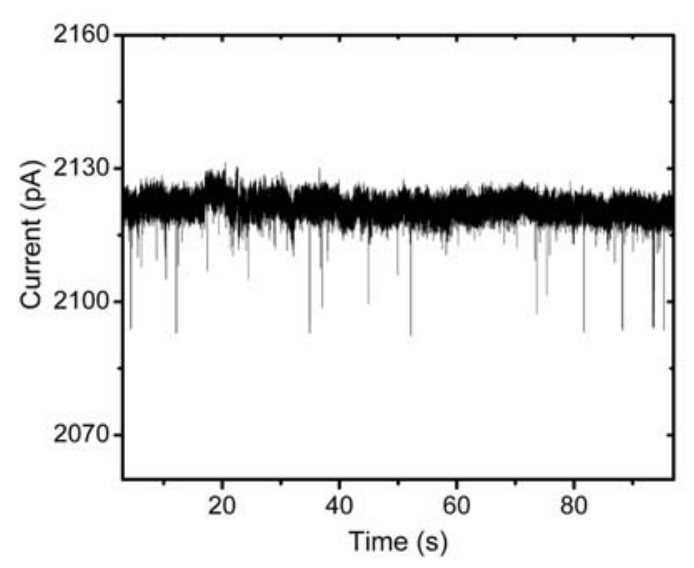

b

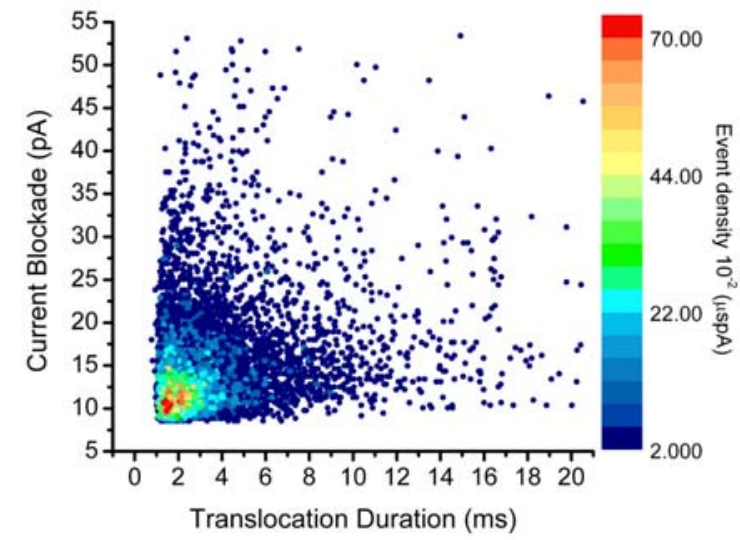

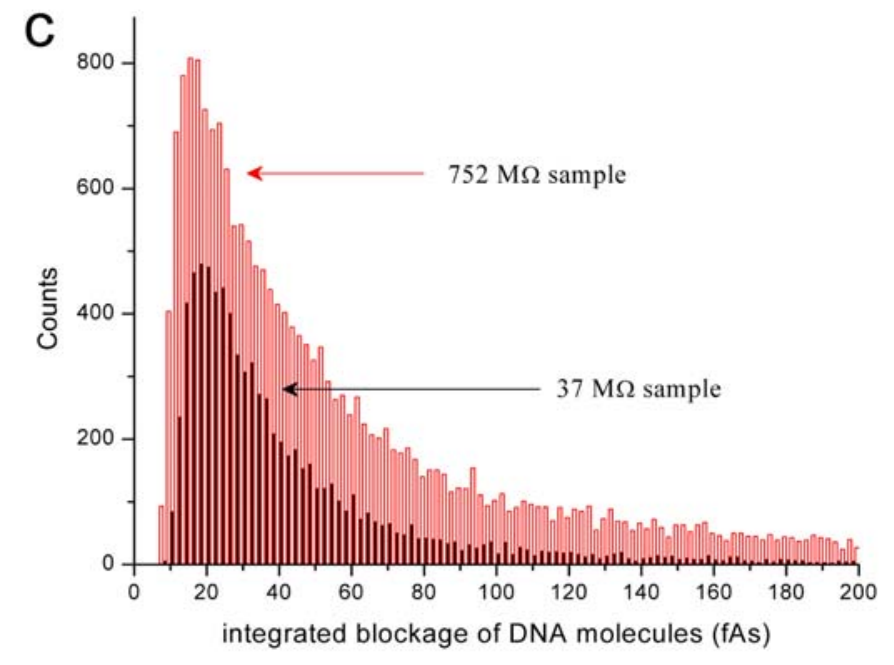

Supporting Figure 2. (a) DNA translocation signals observed with a $37 \mathrm{M} \Omega$ pore, 80 mV bias applied. (b) Density distribution of observed signals: 8,337 events in a single measurement. (c) The integrated area of the blockage current and the translocation duration from both nanopores (37 $\mathrm{M} \Omega$ \& $752 \mathrm{M} \Omega$ ) are shown. Two plots are overlapping over the peaks, which indicate that the same molecules (48 kbp $\lambda$-dsDNA) are recognized in both experiments. 